\title{
Open Source Automation Software: Stirring Automated to Integrated Library System
}

\author{
${ }^{1 *}$ MORUF, HA; ${ }^{2} \mathrm{SANI}, \mathrm{S} ;{ }^{3} \mathrm{ABU}, \mathrm{ZI}$ \\ ${ }^{*}$ Department of Library and Information Science, Federal University Dutsin-Ma, Katsina State, Nigeria \\ ${ }^{2}$ University Main Library, Federal University Dutsin-Ma, Dutsin-Ma, Katsina State, Nigeria \\ ${ }^{3}$ Department of Computer Science, Bayero University, Kano, Kano State, Nigeria \\ *Corresponding author e-mail: hawwau.moruf@yahoo.com, Tel: +234-8022429983
}

\begin{abstract}
The explosion of the World Wide Web; dynamic nature of information technologies, such as open source; the increase in electronic resources; and the rising expectations of library users have contributed to the changing nature of the Automated Library System (ALS) since its inception in the 1970s. These changes are reflected in the conceptual differences between the ALS and the Integrated Library System (ILS). The ALS is identified as simply a database to house and retrieve a library's holdings while ILS is identified as robust clusters of systems involving every process and module related to library operations. This article presents a review on the evolving features of some commonly adopted Open-Source ILS Software (Koha, NewGenLib, Evergreen, PMB and OpenBiblio) which had stirred ALS to ILS, as well as justifications and barriers to the use of open source software in academic libraries.
\end{abstract}

\section{DOI: https://dx.doi.org/10.4314/jasem.v24i7.21}

Copyright: Copyright (C) 2020 Moruf et al. This is an open access article distributed under the Creative Commons Attribution License (CCL), which permits unrestricted use, distribution, and reproduction in any medium, provided the original work is properly cited.

Dates: Received: 16 May 2020; Revised: 29 June 2020; Accepted: 07 July 2020

Keywords: Automation, integrated library system, library, software

The explosion of the World Wide Web, regarded as the Internet; new information technologies, such as open source; the increase in electronic resources; and the rising expectations of library users have contributed to the changing nature of the Automated Library System (ALS) since its inception in the 1970s. These changes are reflected in the conceptual differences between the ALS and the Integrated Library System (ILS). The ALS is identified as simply a database to house and retrieve a library's holdings while ILS is identified as robust clusters of systems involving every process and module related to library operations (Kinner and Rigda, 2009). The main type of software in use in libraries today is the ILS, which are multifunction adaptable software applications that allow libraries to manage, catalog and circulate their materials to patrons. An ILS provides a search interface to the library catalog and automates library tasks such as the tracking of book loans and returns (Riewe, 2008). Although ILS vendors have added many different features, every ILS has nearly the same core components of cataloging and circulation tracking. An ILS usually comprises a relational database, software to interact with that database, and two graphical user interfaces (one for patrons, one for staff). Most ILS(s) separate software functions into discrete programs called modules, each of them integrated with a unified interface (Uzomba et al., 2012). Librarians and programmers have worked together to produce several open source ILS(s). In Open Source Software (OSS), the sequence of humanly readable computer instructions, known as source code, is open to view. Open source licenses ensure that OSS and its derivatives may be freely viewed, used, copied, modified, and redistributed (Open Source Initiative, 2006). In choosing ILS software, libraries must base their decision not only on the performance and efficiency of the system, but also on its fundamental flexibility to readily adapt to the future demands and needs of their patrons (Muller, 2011). There are different types of open-source integrated library system software that have been adopted by various academic libraries in Nigeria. Examples include Koha, NewGenLib, Evergreen and OpenBiblio. A great deal has been written in relation to Open Source library tools, evaluation and impact, however, the focus of this paper is on open source software commonly used for library automation through integrated application of ILS. Edmund (2008) postulates that Koha, Evergreen and $\mathrm{PMB}$ are the most active projects, innovative in areas of Web 2.0 support and are extending the core set of features in their applications. Consequently, this paper explores existing literatures to assess the evolution, features and benefits of five common open 
source automation software that support the stirring of ALS to ILS. It also addresses the justification and barriers to the use of these software in academic libraries.

1. Koha Open Source Software: First deployed in 2000 , Koha is the first open source integrated library system (Reddy and Kumar, 2013) and considered the most successful of the Open Source Software (OSS) ILS projects (Jaffe and Careaga, 2007). The name Koha comes from a Mãori term for a "gift" or "donation" and it is a web-based ILS, with a SQL database (MySql preferred) backend, cataloguing data stored in MARC and accessible via Z39.50 (Macan et al., 2013). Koha user interface is very configurable and adaptable which has been translated into many languages (about 47). It is the most used and this was evident from the finding of Iroaganachi et al. (2015) which revealed that Koha software has gain popularity over the years especially academic libraries in Nigeria.

Features of Koha: The popularity of adoption and usage of Koha in Nigeria libraries could be as a result of its features. Omeluzor et al. (2012) comprehensively states the features of Koha to be flexibility, user-friendliness, easy to use, internet compatibility, web 2.0 features among others. Reddy and Kumar (2013) also indicate that Koha can support Z39.50 search; simple and has clear interface for both librarians and users; and the software has been translated to many languages globally. The study also espouses that Koha supports Web 2.0 facilities like tagging and RSS feeds; the presence of union catalog facility; ability to provide custom search, circulation and borrower management; supports acquisition system like budgeting and pricing information, serials system support; the existence of reading lists, and easy barcode printing, among others. The preceding features of Koha software can be summarized as attributes that change the behaviour of library operations from a one-way flow to a two-way-flow which are characterized by Web 2.0 tools. Library users can now provide feedback based on the information services rendered. However, many libraries use these features as the justification for choosing Koha.

With the release of Koha 3.0 version in 2005 and the integration of the powerful Zebra indexing engine. The software became a viable, scalable solution for libraries of all kinds (http:// www.koha.org). It has most of the features that would be expected in an ILS, including simple clear interface for librarians and members (patrons), union catalog facility, customizable search, circulation and borrower management, serials system for magazines or newspapers among others (Kumar and Raghunadha,
2013). Migration of data from one ILS to Koha can be done easily.

Advantages of Koha: Koha as an open source software is constantly being upgraded to keep pace with new technologies, the advantages of Koha includes:

- No licensing costs: Koha runs on top of an open-source operating system and uses an open-source database management system, which also materializes in a reduction of costs in infrastructure.

Compatibility with any platform: Koha is a $100 \%$ Web-based software solution. The software is centralized on a server, and there is no need to install applications on users' workstations. It requires only a browser to use and access from any device, desktop or tablet.

- Configurable according to the needs of any library.

- $\quad$ Extending services beyond library physical space by providing a set of services that take the library closer to its users.

- $\quad$ Support for library networks: A single Koha instance can support a network of libraries, make each of the libraries to operate independently and according to their own internal policies.

- Koha features (such as OAI-PMH and Z39.50) facilitate the interoperability of information with other library management systems.

- Koha OPAC module promotes the inclusion of all citizens. In accordance with the Web Content Accessibility Guidelines (WCAG 2.0), the system enhances accessibility by implementing a wide range of recommendations to make Web content more accessible to people with disabilities.

- Users can get their data out of Koha later on if they decide to change systems.

2. NewGenLib Open Source Software: NewGenLib was developed as proprietary software in 2005 but in 2008 its version 2.1 declared itself open source software under GNU General Public Licence (Giri, 2012). It is a unique combination of library automation software, digital library software and a database search facilitator. With its version 3.1.1 users can not only search library catalogue, but also search various databases subscribed by the library and some open access databases at a single click. NewGenLib is compatible with International standards such as MARC 21 for bibliographic description, ISBD, OAIPMH Protocol, Z39.50 Protocol, Dublin Core, Unicode, and many more (NewGenLib, 2011).

NewGenLib, unlike Koha is a platform independent software which turns it more beneficial for the user who are not well verse with Linux. NewGenLib has been growing rapidly over the last few years. Its 
unique features and services have attracted libraries all over the world. It is installed in more than 2500 libraries of 58 countries in the world (NewGenLib, 2010).

Features of NewGenLib: Features of NewGenLib open source as gathered by Olatunji et al. (2018) are:

- Licencing: It is open source under the most widely used open source software licensing system called GPL (General Public License).

- $\quad$ Source Code and User Manual: The open source binaries and source code are downloadable. Installation notes for Linux and Windows are also available at the site.

- User's Feedback: The users of the software can post their feedback with views, problems, solutions, discussions, etc to the organization.

- $\quad$ Architecture and Backend: It is web-based and has a multi-tier architecture; it uses Java, the JBoss and PostgreSQL as default backend.

- Functional Modules: Acquisitions management (monographs and serials); technical processing; circulation control; system configuration; a desktop reports application and an end-of-day process (scheduler) application.

Data Create and Exchange Format: NewGenLib open source is compliant with Machine Reaedable Catalogue (MARC-21) format. It has a MARC editor. It allows seamless bibliographic and authority data import into cataloguing templates.

- Mail Server: SMTP mail servers can be configured for emails that can be sent form functional modules.

- Open Access Compatibility: NewGenLib open source allows creation of institutional open access (OA) repositories compliant with the Open Access Initiative

- Unicode Compatibility: NewGenLib open source is Unicode 3.0 compliant.

- Radio-frequency identification (RFID) Technology: It is RFID ready.

Advantages of NewGenLib: The advantages of NewGenLib open source may be perceived as follows in the light of the advantages of open source software as pointed out by Richard (2008):

- $\quad$ Ability to tailor to fit local needs: The availability of the source code means that a user can modify and enhance the software to more closely fit its own needs.

- No restriction on use: unlike commercial software, there are no contractual restrictions on how the software is used. While some developers use the GNU General Public License that assures users that they have the right to distribution and those to whom they distribute also have the right to modify and distribute, other developers merely declare that their software is in the public domain. A subsequent user may, therefore, decide to protect the enhancements that it makes by copyrighting them.

Low cost: There is no charge for the software; therefore, the capital outlay required by commercial software is avoided. The major costs are ongoing development and maintenance. If the number of users is large, and they share their efforts, each user's cost is reduced. However, if the number is small or a user does a lot of tailoring to fit unique local needs that are not shared by other users, the cost can escalate.

However, some of the disadvantages of this type of open source software are: lack of coordination, inadequate training and technical support, lack of participation, lack of guarantees and remedies, scalability and speed etc. The open source software may not offer the scalability and speed of proprietary software because the easy-to-use and general-purpose programming languages used are not very scalable and are slower than other languages. But recently, NewGenLib open source has overcome this problem. On the other hand the Versus Solution Pvt. Ltd organizes workshop and training programmes for appropriate support.

3. Evergreen Open Source Software: Evergreen is an open source Integrated Library System (ILS) initially developed by the Georgia Public Library Service for Public Information Network for Electronic Services (PINES), a state-wide resource-sharing consortium with over 270 member libraries (Weber, 2006). Beyond PINES, the Evergreen ILS is deployed worldwide in approximately 1,800 libraries, and is used to power a number of state-wide consortia catalogs (Breeding, 2007). Evergreen was developed because of the limitations of the ILS. It is constantly being developed and improved on as is available to any library who wants it for free. Development priorities for Evergreen are that it be stable, robust, flexible, secure, and user friendly (Fox, 2007).

\section{Features of Evergreen}

- Circulation: for staff to check items in and out to patrons

- Cataloging: Evergreen is known for an extremely flexible indexing system that allows for a high level of customization and by default uses Library of Congress MODS as its standard.

- Online Public Access Catalog (OPAC): a public catalog, or discovery interface, for patrons to find and request books, view their account information, and save book information in Evergreen "bookbags". The OPAC received a makeover in early 2009 with the new, optional skin, Craftsman. There is 
also an optional Children's OPAC. Various patron services such as paying bills by PayPal and Stripe, optional retaining of circulation history, book bags etc.

- $\quad$ Self Service - Evergreen comes with selfcheckout and registration options that can be activated by the libraries.

- $\quad$ Acquisitions: For staff to keep track of those materials purchased; invoices, orders etc.

- $\quad$ Statistical Reporting: powerful reporting for retrieval of any statistical information stored in the database.

- SIP 2.0 supports: For interaction with computer management software, self-check machines, and other applications.

Advantages of Evergreen OSS: Being one of the newest open source applications, its advantages include;

- It has code and schema design which include strong OOP philosophy.

- It has a large network installed base, including the Georgia Library PINES network of over 200 libraries.

- $\quad$ Evergreen, like Koha, has a CGI (Common Gateway Interface) mode of server operation, but has clearly been able to support the PINES network.

4. PMB Open Source Software: PMB (formerly known as PhpMyBibli) is a very functional ILS, with all necessary basic functions of library automation especially with regard to:

- Integration of Web 2.0-oriented features and other patron-based Web services: (review of documents, add comments and tags from users);

- $\quad$ Federated search connectors for Z39.50 and

OAI;

- $\quad$ Safeguarding the security of all data and full system restore from the safety backup;

- $\quad$ The creation of item records on the fly for documents with no bar code.

PMB provides options for SDI (Selective Dissemination of Information Service), reindexing, an interface for database back up and import/export of bibliographical records, the determination of default values, choice of interface languages (9 languages), choice of interfaces and friendly Web-based OPAC (Muller, 2011). PMB also has, in addition to a default report generator, a module to generate specific reports. It is thus possible to query all data together, but this requires a basic knowledge in SQL query language, which may be a limiting factor for library staff.

PMB has functional richness with the following advantages:

- Installation is relatively simple because of its internationalisation support features.
- It has gone further in exploring Web 2.0 features.

- It has a PHP/MYSQL base and good use of classes for code reuse.

- It is the most elegant of the current offerings for a small multi-library network setting.

- It has largely been implemented in European libraries, and is strongest in its French support base. Although it is multilingual in template design, however, strengthening of implementation examples in English will improve its adoption outside Europe continent.

5. OpenBiblio Open Source Software: OpenBiblio is popular with small and rural libraries worldwide due to its simplicity, extensive language support, and good documentation (Kamble et al., 2012). Openbiblio was created in 2002 by Dave Stevens, who was interested in creating an easy-to-use, well-documented, easy-toinstall library system (Zargaryan, 2011). Though the system is still under active development, it has already become widely used in small libraries and archives worldwide.

\section{Features of OpenBiblio}

- $\quad$ Circulation: for staff to check items in and out to patrons, and to add new patrons.

- $\quad$ Cataloguing: for staff to create, modify, or delete bibliographic records, including uploading of MARC and MARCXML records.

- Online public access catalog (OPAC): a public catalog for patrons to find books

- Administration: Configuration and management of the system, including library, staff, material, fines, and website settings.

- Reports: Retrieve and format information from the database, including overdue letters and statistical models for the use of the library's materials. OpenBiblio uses a special syntax called RPT for its reports, so that users do not have to learn PHP to create these reports.

Advantages of OpenBiblio includes; It is popularly used and suitable in small libraries and It is known for simple installation and effective presentation. While OpenBiblio provides all the essential functionalities for a small or medium-sized library, it does not include the more complex features, such as acquisitions and serials management, provided by other open source integrated library systems, such as Koha or Evergreen (Kumar, 2005).

Justifications for the use of open source software in libraries: With the wide acceptability and adoption of open source software, automated library system is undergoing significant changes to integrated library 
system in many libraries of the world, including developing country like Nigeria. Wheeler (2007) stated that "open source software gives users the freedom to run the program for any purpose, to study and modify the program, and to redistribute copies of either the original or modified program without having to pay royalties to previous developers". Ukachi (2012) stressed that "the major reason to choose an OSS application is the freedom it confers to change the source code for individual requirements.

Gonzalez-Barahona (2000) enumerated the following justifications for open source software:

- Auditability: software with published source code. Without access to the source, third party inspection is impossible. By publishing the source code, authors make it possible for users of the software to have confidence that there is a basis for those claims.

- Cost: Most Open Source software are provided free of royalties and fees. Administrative overhead cost is drastically minimal as there is no cost attached to number of copies in use, unlike when proprietary software is used. There is also lower management cost as no upgrade fees are incurred.

- Flexibility and freedom: This software is flexible as it gives users opportunity to be able to choose solutions suitable for their needs. Open source software offers its users greater freedom to purchase other products, avoiding lock-in to particular manufacturers, freedom from a single vendor and freedom to modify your software.

- Reliability: Open source software could be said to be reliable because it does not manifest defects which can cause incorrect operation, data loss, sudden failures, or failure to meet specification or appropriate published standards which is generally termed as 'bug'. This is not to say that problems are never encountered with the use of OSS but, each problem is usually addressed with speedy fixes, a process which is undoubtedly assisted by the availability of the source code.

- Stability: Having access to the source code can allow a business to choose to support itself on an old version where necessary thereby giving more options and choice to the users.

Barriers To The Use Of Open Source Software In Libraries: Though open source software are considered to be best option for libraries, however there are some issues that are related to its adoption and usage (Uzomba et al., 2015). Some of these barriers are mentioned below:

- Data migration: Most libraries have this fear of losing data in the process of migrating from their existing practice to open source option because of its perceived technicalities.
- Inadequate power supply: In developing country like Nigeria, electric supply to libraries is not stable. Software requires servers and network infrastructure to function and this could be hinder by poor power supply.

- $\quad$ Lack of proper documentation: The lack of proper documentation inherent in open source software makes it very difficult to attend to end-users' questions and overcome unexpected challenges that may arise.

- $\quad$ Lack of support: The available support for open source software is predominantly self-motivated discussions found on the Internet, and since the software is constantly being changed, no manuals or instructions are made. Where there is no vendor responsible for the software, support for open source applications can vary, and often depends on the user/developer community's commitment to the project (Chawner, 2004).

- $\quad$ Lack of technical knowledge from the staff: some libraries choose proprietary software because they lack the necessary technical skills to support open source in-house (Clarke, 2000).

- $\quad$ Poor funding: Many libraries, especially in developing countries are poorly funded. Libraries always complain of inadequate funds to train their staff on how best to develop and handle modern facilities needed for the open source initiative.

Other setback to open source movement as enumerated by Uzomba et al. (2015) includes: Cost of procurement of the hardware/software, lack of consortium, lack of training and re-training of staff, crashing problem and maintenance cost.

Conclusion: Among the several open source options available for libraries, Koha has been identified to be viable, scalable solution for libraries of all kinds; NewGenLib has become very competitive to Koha while Evergreen and PMB possess basic functions of library automation. OpenBiblio is suitable for use in small libraries because it is easy to install, customize and use. The availability, affordability and flexibility of open-source software has made the stirring of Automated Library System to Integrated Library System possible in our academic libraries.

\section{REFERENCES}

Breeding, M (2007). Next-generation flavour in integrated online catalogs. Lib. Tech. Rep. 43(4): 38-41.

Chawner, B (2004). Free/Open Source Software: new opportunities, new challenges. $12^{\text {th }}$ biennial VALA conference and exhibition, 3-5 February 2004, Melbourne Convention Centre: conference proceedings. 
Clarke, KS (2000). Open software and the library community. M.I.L.S. research paper, School of Information and Library Science, University of North Carolina - Chapel Hill, Chapel Hill, NC.

Edmund, B. (2008) Open Source Library Management Systems: A Multidimensional Evaluation, Aust. Acad. Res. Lib. 39:1, 1-13

Fox, R (2007). Digital libraries: The systems analysis perspective: The great pig roast. OCLC Sys. 23(3): 242249.

Giri, R (2012). NewGenLib 3: an integrated open source library management system that makes your library visible in web. Lib. Hi Tech New. 29(10): 4-12.

Gonzalez-Barahona, JM (2000). Advantages of Open Source Software. Available: http://eu.conecta.it/paper/advantages.html.

Iroaganachi, MA; Iwu-James, J; Esse, U. C (2015). Software selection and deployment for library cooperation and resource sharing among academic libraries in SouthWest Nigeria. DESIDOC J. Lib. Info. Tech.35(1): 3-8.

Jaffe, LD; Careaga, G (2007). Standing up for open source. Lib. Phi. Prac. 9(3): 1-17.

Kamble, VT; Hans, R; Sangeeta, J (2012). Open Source Library Management and Digital Library Software. DESIDOC J. Lib. Info. Tech. 32 (5): 388-392.

Kinner, L; Rigda, C (2009). The Integrated Library System: From Daring to Dinosaur. J. Lib. Admin. 49: 401-417.

Kumar, K; Raghunadha, RT (2013). Analytical study on knowledge about open source software in technological institutional LIS professionals. Inter, J. Lib. Info. Sci. 5(11): 439-446.

Kumar, V (2005). Free/Open source integrated library management systems: comparative analysis of Koha, PHPMyLibrary and OpenBiblio. University of Calicut.

Macan, B., Vanesa, F., G. \& Stojanovski, J. (2013). Open source solutions for libraries: ABCD vs Koha. Prog. 47(2): 136-154.

Muller, T (2011). How to choose a Free and Open Source Integrated Library System. Inter. dig. Lib. Pers. 27(1): 57-78.

NewGenLib (2010). NewGenLib News. Retrieved March 28, 2015, from http://www.verussolutions.biz/digitalLibrary.php.

NewGenLib (2011). Standard based. Retrieved April 5, 2013, from http://www.verussolutions.biz/web/content/standardsbased.
Olatunji, SO; Farouq, BL; Idris, M (2018). Adoption of Integrated Library Management Software (NewGenLib): The Experience of Kano University of Science and Technology Library, Wudil, Kano State" (2018). Lib. Phi. Prac. (e-journal) 2140.

Omeluzor, SU; Bamidele, IA; Ukangwa, CC; Amadi, HU (2012). The relevance of a library in the 21 st Century: Students perception. Inter. J. Lib. Info. Sci. 5 (6): 160167.

Open Source Initiative. (2006). The open source definition. Retrieved October 1, 2006, from http://www.opensource.org/docs/definition.php.

Reddy, TR; Kumar, K (2013). Open Source software and their impact on library and information center: AN overview. Inter. J. Lib. Info. Sci. 5(4): 90-96.

Riewe, LM (2008). Survey of open source integrated library systems. MLIS Thesis, San Jose State University. Pp 108.

Ukachi, B (2012). Awareness, availability and utilization of open sources software in Nigerian Libraries: the way forward. Inter. Res. J. Lib. Info. Arch. Stu. 2 (1): 001009.

Uzomba, EC; Oyebola, OJ; Izuchukwu, AC (2015). The use and application of open source integrated library system in academic libraries in Nigeria: Koha Example. Lib. Phi. Prac. (e-journal) 1250.

Wallace, PM (1991). Gary M. Pitkin (ed.). Library Systems Migration: An Introduction. Westport, CT: Meckler. pp. $1-7$.

Weber, J (2006). Evergreen: Your Homegrown ILS-An inhouse team uses open source technology to compete successfully with commercial vendors in the library automation sphere. Lib. J. 131(20): 38-41.

Wheeler, DA (2007). Why Open Source Software / Free Software (OSS/FS, FLOSS, or FOSS)? Look at the Numbers! http://www.dwheeler.com/contactme.html.

Zargaryan, T (2011). OpenBiblio in Armenia: Automation of Small Libraries. (http://lists.eifl.net/system/files/201201/eiflfosscasestud y2011 openbiblioarmenia.doc). 